\title{
Application Status and Design Requirements of Virtual Reality Technology in Dance Teaching
}

\author{
Lusi Huang \\ School of Art, Quanzhou Preschool Teachers College, Quanzhou Fujian, 362000, China
}

Keywords: Virtual Reality, Dance Teaching, Application Status, Design Requirement

\begin{abstract}
China has continuously pursued culture innovation and culture construction in recent years. Dance culture also belongs to a part of culture construction. At present, certain gap in culture construction still exists between China and Western countries. An obvious gap is in dance teaching technology. The comparison of the application status of virtual reality technology in dance teaching at home and abroad is conducted in this paper. Then, the design requirements of virtual reality technology in dance teaching are expounded. Finally, relevant suggestions are proposed to enlighten the peers.
\end{abstract}

\section{Introduction}

Virtual Reality (VR) technology is a teaching means which has been praised highly in the educational circle in recent years. It combines virtuality and reality and plays a promotion role in education reform. Its characteristics include immersion, imagination and interactivity which quite meet the requirements of new curriculum reform. The blind explanation in traditional teaching will let students feel boring and dull. VR technology can make students dialogue with the ancients while appreciating their poems in classroom, which breaks time and space limits. Besides, VR technology can avoid certain dangers. For example, it can give play to its unique functions in earthquake and fire drilling. The application of VR technology in dance teaching can solve such problem that students are not personally on the scene in dance rehearsal and lack certain emotional resonance. Virtuality and reality can be combined. Emotion and dance can be combined to let the beauty of dance shown more vividly.

The appearance of VR technology makes dance teaching combined with VR technology. In particular, it exerts its characteristic of immersion. After wearing the helmet or glasses, students can generate the feeling of being dancing in the scene in dance learning process. For example, during dancing The Fern Leaf Hedge Bamboo in the Moonlight, students will have the feeling of dancing in front of the stilted building after wearing the helmet. When dancing The State of Ceremonies, students will have the feeling of wearing beautiful dress and dancing on the grand banquet......

\section{Application Status of VR technology in Dance Teaching}

VR technology is introduced from abroad. Foreign VR technology is more advanced than China's VR technology. Next, comparison and analysis of application status of VR technology in dance teaching are conducted.

\subsection{Foreign Application Status}

At first, VR technology was used for virtual training of boxing abroad. It can show the position of the opponent in the form of optical wave so that the boxers can train their reaction capacity and execution ability. Later, VR technology started to promoted. VR technology attracts numerous audiences due to its peculiarity and is welcomed by the market (especially in boxing training). The application of VR technology in boxing training can enhance human-machine interestingness and avoid certain harm. It is suitable for initial boxing training. This is the basis of VR technology 
application in dance teaching. Many investors overseas invented corresponding video clip scope for promotion of VR technology and corresponding devices. For instance, Sogary designed a modern dance learning system, and helped students without any foundation learn dance by video teaching movement capture feedback system. Dahl Saif invented a dance teaching system with 3D animation, text explanation and video switchover. People can browse information while watching TV, and then shift the picture continuously in their mind. The teaching system is convenient and fast and reaches the good teaching effect. There are many such examples. The application of VR technology abroad is much wider than that in China. Next, we will introduce the application status of VR technology in China.

\subsection{Domestic Application Status}

Domestic application of VR technology in dance teaching is still in the initial and development stage. The major achievements of three figures are mainly introduced as follows. Chen Qixiang studied VR technology on the basis of Bianzhong dance and was praised by people. Guo Jin et al. researched robot dance and utilized VR technology to design simulated skeleton dance model of 3D virtual robot by grasping the dancing rules of robot. The virtual dance visual system designed by Chen Fang can be used in dance making and arrangement. Domestic application of VR technology is being enhanced, and there will be many new breakthroughs in the future.

\section{Design Requirements of VR Technology in Dance Teaching}

The application of VR technology in dance teaching is in the gradual development stage. To develop it better, it is required to adopt the strengths, avoid the shortcomings, continuously innovate and combine virtuality and reality so as to reach the better teaching effect. So, the design requirements of VR technology in dance teaching will be staged next.

\subsection{Thorough Composition System of VR Technology}

To make the design of VR technology in dance teaching more complete, a thorough composition system of VR technology is required. The composition system should include picture, scene creation, $3 \mathrm{D}$ design, movement capture system and movement correction system. The picture aims to let students take a panoramic view of the situation more vividly at first and roughly grasp the dance content. Scene creation utilizes immersion and imagination of VR technology, and let students seemingly be personally on the scene and add their emotions so as to make the dance more beautiful. Only when students can put their emotions in the dance can the stage effect of the dance be better and more unique. Movement capture system aims to let students feel whether their dance movements are correct. Then, the movement capture system reflects the summary into the teacher's helmet. The teacher may arrange students to take dance training with different intensity according to students' dance movement rehearsal. Except movement capture system, movement correction system is also required. Movement correction system can remind students of inaccurate movements in the form of radio wave and help them correct their dance movements. Such movement correction system can greatly improve the teaching efficiency of dance teachers and help dance teachers relieve teaching burden. In this way, the teachers need not observe students and correct their movements anytime. The teachers may focus on the severe mistakes and pay more attention to dance arrangement.

We will take Quanzhou Liyuan opera as the example for teaching. With long-standing mentoring relationship, Quanzhou Liyuan opera spreads by oral imparting with physical instruction such as "saluting by cupping hands to the chin, separating hands to the belly button, and raising hands to the eyebrow", "fingers and hands to the nose, partially touching both ears and lifting hands to the breast", which are pithy formulas that are inherited familiarly and repeatedly generation by generation. However, due the large quantity, "Kebu" and "Kemu" are usually confused in detailed items of numerous opera genres, which has brought great trouble to teachers' instruction and made students feel hard in opera practice. But it is because of the unique and rigorous performance rules of "Shiba Kemu" that create the elegant and graceful style of Liyuan opera. Also, the so-called "Shiba Kemu" is 
just a general term. Thus, it is the priority among priorities to help students proficiently apply these skills of Liyuan opera performance art in teaching process. The application of virtual reality technology can bring an immersive feeling for students and provide new transmission channel for students by experiencing the inner actions. It can help students learn the opera in an understanding and experiencing oriented environment, and greatly improve their action practice efficiency. Meanwhile, the action correction system can effectively solve the problem that teachers easily get bored during the teaching process.

\subsection{Functional System of VR Technology in Dance Teaching}

We have introduced the composition system of a thorough VR technology above, roughly including four aspects. Now, we are about to introduce the design requirements of VR technology in dance teaching for the requirements of functional system. The function should be strong to support teaching activity implementation, so functional system should be expounded form the following three aspects:

\subsubsection{Dance Exhibition}

In Traditional Dance Teaching, we often adopt video teaching watching or teacher's demonstration. When VR technology is applied in dance teaching, we can also utilize 3D picture to display the dance content except watching dance teaching vide through multimedia screen. VR helmet or VR glasses can let students see the decomposed movements and correct demonstration of each part at all directions. Teacher's personal demonstration may be transformed to from the original video dance teaching so that students can have the feeling of familiarity. In this way, students can master the dance content within the shortest time.

\subsubsection{Movement Capture}

The movement capture system is mentioned above. Here, the movement capture system is explained in detail. The movement capture system shoots students' movements through the helmet or glasses, and then feeds back the movements to the teachers so that teachers can roughly grasp the dance rehearsal situation. Then, the teachers may adjust students' rehearsal intensity and rest time according to their rehearsal situation.

\subsubsection{Effect Feedback}

Effect feedback is a humanized system and it is also a system to test dance rehearsal results. It pays more attention to summarizing dance teaching effect and then feeds it back to the large screen. The movement capture system may be used to shoot students' rehearsal situation and analyze the effect. Then, the system feeds the result to students' helmet and glasses so that they can see their performance and scores, cognize their shortcomings, adopt others' strong points and avoid their own weaknesses. Such effect feedback system is more scientific and fair than teacher's comments and criticism. For students, such feedback effect is more persuasive. For teachers, such effect feedback system greatly improves teaching quality and lightens teacher's teaching burden. Liyuan opera Kebu learning that we have listed hereinbefore is a typical example that using effect feedback to realize the combination of Quanzhou Liyuan opera traditional teaching and virtual reality technology. So here no more detailed description will be provided.

\section{Suggestions on Application of VR Technology in Dance Teaching}

\subsection{To Enhance Learning of VR Technology}

VR technology was not introduced in China long, so its research is limited. Teachers and students need to enhance the study of VR technology. Due to the limits of teaching conditions, time and space, technological progress can let us break these limits. Hence, many developed areas start to introduce VR technology to carry out teaching reform. To keep up pace the era development, dance teachers need to enhance the study of VR technology, grasp the skills of VR technology, continuously 
innovate and explore unique teaching modes or new inventions. Enhancing the study of VR technology means higher requirements for dance teachers. The era advances, and dance also should advance with the era. VR technology cannot just know the ancient events and the modern events, but also look into the future. VR technology can be used to arrange more dance programs so that common people have the impressive feeling.

\subsection{To Properly Utilize VR Technology in Training}

The second suggestion on the application of VR technology in dance teaching is to properly utilize VR technology in training. Technological progress may easily result in human laziness and inert thought. A typical example of applying VR technology in training is Quanzhou Liyuan opera Kebu. Kebu derives from religious culture, real life and animal action (bionics). Kebu exists in art culture as an imitation art, and is favored by the audiences. Liyuan opera Kebu is different, but the majority is Gongshou, Fenshou and Jushou... Kebu involves many contents, so certain difficulty exists in dance teaching and dance learning. The appearance of VR technology can greatly solve this difficulty. Hence, it is required to properly apply VR technology in training. Its advantages may be used for dance teaching and dance learning. During learning Kebu, movement capture effect feedback system of VR technology may be utilized for teaching and guidance. The application of VR technology can gain the effect of getting double results by doing half work.

\section{Conclusions}

In conclusion, the application of VR technology in dance teaching can help dance teachers improve teaching effect and let students' conduct dance training without teachers. Modern technology is utilized to guide students' dance learning. Dance teaching demonstration, movement demonstration and feedback are shown in VR form, so VR technology is favored by many teachers and students. In current dance teaching in China, the advantages of VR technology should ne continuously applied to develop China's dance culture and conduct stronger culture construction. The powerful functions of VR technology should be applied to improve dance construction content and let China's dance cause develop better.

\section{Acknowledgements}

This research was financially supported by the 2016 topic in "the 13th Five-year Plan" Dance Performance Form and Teaching Value of Quanzhou Liyuan Opera Kebu (FJJKCG16-371).

\section{References}

[1] Ji Wenjun, Study on Interaction Design of VR Animation, Central Academy of Fine Arts, 2017.

[2] Azimkulov Ayan, Research on Virtual Teaching of VR Technology, Donghua University, 2017.

[3] Liu Dejian, Liu Xiaolin, Zhang Yan, Lu Aofan, Huang Rongbei, The Application of Virtual Reality Technology in Education: Potential, Current Situation and Challenges, Open Education Research, 2016,22(04):25-31.

[4] Li Xiaopeng, Application Status and Design Requirements of VR Technology in Dance Teaching, Home Drama, 2015(22):191.

[5] Liu Dong, Sun Huijia, the Application and Design Requirements of Virtual Reality Technology in Dance Teaching, China Educational Technology, 2014(06):85-88.

[6] Zha Yan, Research on Application of Digital Media Technology in Sports Dance Teaching, Dalian University of Technology, 2014. 